\title{
Charge-Noise Insensitive Chiral Photonic Interface for Waveguide Circuit QED
}

\author{
Zhang, Yu Xiang; I Carceller, Carles R.; Kjaergaard, Morten; Sørensen, Anders S.
}

Published in:

Physical Review Letters

Link to article, DOI:

10.1103/PhysRevLett.127.233601

Publication date:

2021

Document Version

Publisher's PDF, also known as Version of record

Link back to DTU Orbit

Citation $(A P A)$ :

Zhang, Y. X., I Carceller, C. R., Kjaergaard, M., \& Sørensen, A. S. (2021). Charge-Noise Insensitive Chiral Photonic Interface for Waveguide Circuit QED. Physical Review Letters, 127(23), [233601].

https://doi.org/10.1103/PhysRevLett.127.233601

\section{General rights}

Copyright and moral rights for the publications made accessible in the public portal are retained by the authors and/or other copyright owners and it is a condition of accessing publications that users recognise and abide by the legal requirements associated with these rights.

- Users may download and print one copy of any publication from the public portal for the purpose of private study or research.

- You may not further distribute the material or use it for any profit-making activity or commercial gain

- You may freely distribute the URL identifying the publication in the public portal 


\title{
Charge-Noise Insensitive Chiral Photonic Interface for Waveguide Circuit QED
}

\author{
Yu-Xiang Zhang $\odot,{ }^{1,{ }^{*}}$ Carles R. i Carceller $\odot,{ }^{2}$ Morten Kjaergaard, ${ }^{3}$ and Anders S. Sørensen $\oplus^{1}$ \\ ${ }^{1}$ Center for Hybrid Quantum Networks (Hy-Q), The Niels Bohr Institute, University of Copenhagen, \\ Blegdamsvej 17, 2100 Copenhagen Ø, Denmark \\ ${ }^{2}$ Department of Physics, Technical University of Denmark, Fysikvej 307, 2800 Kongens Lyngby, Denmark \\ ${ }^{3}$ Center for Quantum Devices, Niels Bohr Institute, University of Copenhagen, 2100 Copenhagen $\emptyset$, Denmark
}

(Received 6 July 2021; accepted 22 October 2021; published 29 November 2021)

\begin{abstract}
A chiral photonic interface is a quantum system that has different probabilities for emitting photons to the left and right. An on-chip compatible chiral interface is attractive for both fundamental studies of lightmatter interactions and applications to quantum information processing. We propose such a chiral interface based on superconducting circuits, which has wide bandwidth, rich tunability, and high tolerance to fabrication variations. The proposed interface consists of a core that uses Cooper-pair boxes (CPBs) to break time-reversal symmetry, and two superconducting transmons that connect the core to a waveguide in the manner reminiscent of a "giant atom." The transmons form a state decoupled from the core, akin to dark states of atomic physics, rendering the whole interface insensitive to the $\mathrm{CPB}$ charge noise. The proposed interface can be extended to realize a broadband fully passive on-chip circulator for microwave photons.
\end{abstract}

DOI: 10.1103/PhysRevLett.127.233601

The emission of photons from quantum systems is typically nonchiral, meaning that the emitted photons have equal probability to propagate in opposite directions. The opposite situation, where the emitted photons propagate in a single direction, allows for a wide range of novel phenomena including cascaded driven-dissipative dynamics [1,2], spin dimers [3,4], photonic bound states [5-7], and solvable models of waveguide quantum electrodynamics (QED) [8]. Chiral interfaces enabling the study of such effects have recently been developed for optical photons [9], but optical systems are prone to losses, limiting the quality of the interfaces. In comparison, superconducting circuits realize almost ideal interfaces to guided microwave photons [10-12], potentially allowing for much cleaner demonstrations of these fundamental effects, but so far no chiral interactions have been realized for these systems.

Chiral interfaces to superconducting systems are also of immense technological interest. Scaling superconducting quantum computers to larger sizes puts high demands on the ability to route signals between different components on the chips, e.g., using circulators. Such circulators necessarily break Lorentz reciprocity and thereby time-reversal symmetry (TRS). Commercially available circulators often exploit the Faraday effect [13]. However, such components are off-chip, requiring additional space inside the experimental apparatus. Most of the existing proposals for on-chip circulators break TRS by tailored active control [10,14-23], e.g., via a synthetic magnetic field [22], or by dynamically modulating switches and delays [23]. On-chip circulators that can be operated in a passive form, thereby simplifying its experimental implementations, however remain elusive. A Josephson junction ring threaded by a constant magnetic flux breaks TRS [24], but its applicability as a circulator in current superconducting hardware is challenging, because the system must be operated in the Cooper-pair-box (CPB) regime [24-26] which is susceptible to charge noise [11,27] and limited by bandwidth and fabrication variations [28]. The bandwidth can, however, be increased substantially by exploiting carefully engineered circuits with high impedance [29].

In this Letter, we develop an on-chip tunable broadband charge-insensitive chiral system illustrated in Fig. 1(a). The system is designed such that the (nondegenerate) excited states decay by only emitting photons in one direction, left or right. It has a core composed of two CPBs and an applied external flux (green box), and two transmons that connect the core to the waveguide at two separated locations, being reminiscent of a "giant atom" [30-32]. The core is essential for breaking TRS, while the transmons amplify the coupling to the waveguide and hence the bandwidth. Furthermore, by operating in a particular dark state configuration [33] we show that excitation of the CPBs can be suppressed, such that the interface maintains the excellent coherence properties of transmons [34,35], while still exploiting the inherent breaking of TRS by the CPBs. The interface can directly be extended to realize a circulator for routing signals in large scale superconducting devices. Finally, the tunability of our chiral interface allows compensating fabrication variations as well as control of the operating frequency within a range of a few $\mathrm{GHz}$.

Recent related proposals [36,37] have used giant atoms formed by transmons and careful adjustments of parameters to realize unidirectional interactions with two degenerate levels, which decay in opposite directions. This degeneracy 


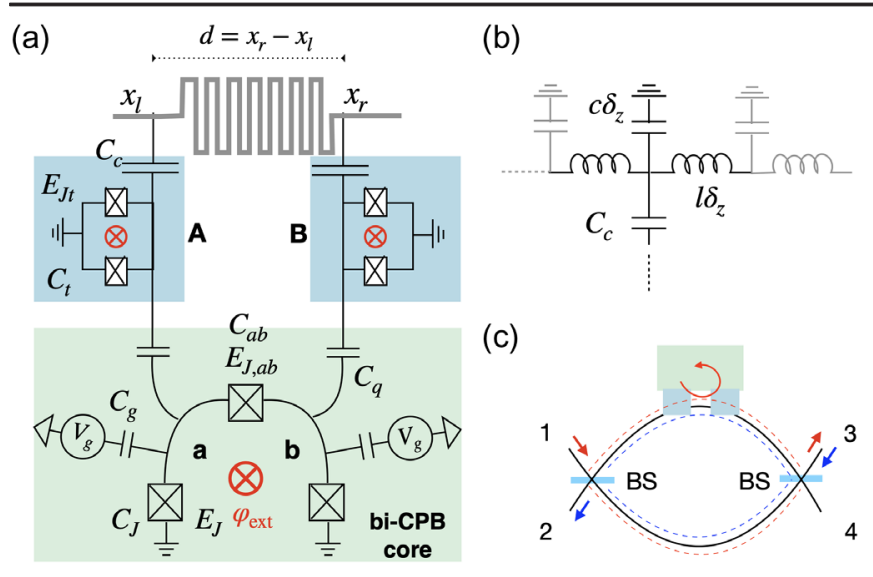

FIG. 1. Proposal for realizing the on-chip compatible chargenoise insensitive chiral photonic interface. (a) Circuit of the chiral interface consisting of two CPBs (green boxes) coupled to a waveguide (top) through two tunable transmons (blue boxes) connecting to the waveguide at positions $x_{l}$ and $x_{r} . C_{J}$ and $E_{J}$ denote the capacitance and Josephson energy of the CPBs, $C_{a b}$ and $E_{J, a b}$ are those of the junction connecting CPB- $a$ and CPB- $b$, $\varphi_{\text {ext }}$ is the dimensionless external flux, $C_{g}$ is the gate capacitance, $C_{q}$ couples the $\mathrm{CPB}$ and transmons, $C_{t}$ and $E_{J_{t}}$ are the total capacitance and (tunable) Josephson energy of the transmons and $C_{c}$ couples the transmons to the transmission line. (b) Circuit model of the capacitive coupling between a transmon and the transmission line. (c) A circulator based on chiral interface (righthand chirality) and Mach-Zehnder interferometer. "BS" denotes 50:50 beam splitters. Input from port 1 goes to port 3 while input from port 3 goes to port 2 .

means that TRS is not broken, and the additional level provides a complication of the dynamics, in particular, for nonperfect or partially chiral systems. Compared to these works, our chiral interface realizes the paradigmatic chiral model of a two-level system with chiral coupling, has tunable operating frequency, and more importantly, can be used for photonic circulators. During the final stages of this work, a different proposal suggested a chiral interface based on a specially designed waveguide and active driving [38].

Preliminaries.-We first consider two coupled CPBs, " $a$ " and " $b$," which as a whole, has the ground state $\left|g_{a b}\right\rangle$ and a nondegenerate excited state $|e\rangle$. The transition between them is resonant with waveguide modes of wave number $\pm k_{0}$. We couple CPB- $a$ and CPB- $b$ to the waveguide at positions $x_{l}$ and $x_{r}$, respectively [see Fig. 1(a)], resulting in coupling operators of the form $\hat{a}_{ \pm k_{0}}\left(\hat{n}_{a}+e^{ \pm i k_{0} d} \hat{n}_{b}\right)$, where $\hat{a}_{ \pm k_{0}}$ is the photon annihilation operator, $d=x_{r}-x_{l}$ and $\hat{n}_{a / b}$ is the CPB charge number operator. Within the rotating-wave approximation and perturbation theory, the decay from $|e\rangle$ to left-right going photons depends on the magnitude of

$$
\lambda_{ \pm}=\eta_{a}+e^{ \pm i k_{0} d} \eta_{b}, \quad \eta_{a / b}=\left\langle e\left|\hat{n}_{a / b}\right| g_{a b}\right\rangle .
$$

This formula expresses the interference between excitation decaying through the left and right arms in Fig. 2(a), which
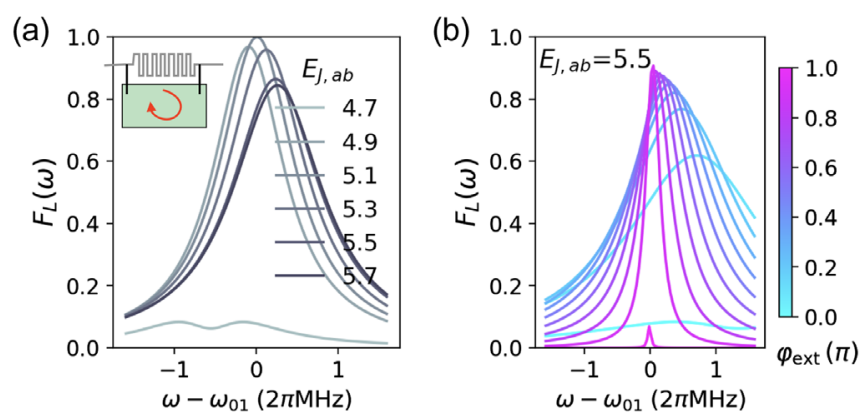

FIG. 2. Fidelity of circulator $F_{L}(\omega)$ against the detuning to the first excited state for direct coupling between the waveguide and the bi-CPB core. The device parameters are $c \delta_{z}=C_{q}=C_{\Sigma}=$ $C_{a b}=1 \mathrm{fF}, E_{J}=5.5 \mathrm{hGHz}$, and (a) $\varphi_{\text {ext }}=0.5 \pi$ and various values of $E_{J, a b}(h \mathrm{GHz})$; (b) $E_{J, a b}=5.5 \mathrm{hGHz}$ and the external flux $\varphi_{\text {ext }}$ varying from 0 to $\pi$.

depends on both the coupling matrix element $\eta_{a / b}$ at $x_{l / r}$ and the phase $e^{ \pm i k_{0} d}$ from waveguide propagation between them.

A chiral interface with a single excited state requires TRS to be broken since left and right propagation are connected by TRS, and a state invariant under TRS cannot distinguish these two possibilities. Breaking of TRS, however, may induce a phase difference between $\eta_{a}$ and $\eta_{b}$ allowing the cancellation of $\lambda_{+}$or $\lambda_{-}$for a suitable $k_{0} d$ if $\left|\eta_{a}\right|=\left|\eta_{b}\right|$.

Incorporating the chiral interface into one of the arms of a balanced Mach-Zehnder interferometer enables the construction of a four-port photonic circulator [Fig. 1(c)]. Suppose the scattering matrix of the chiral interface is $S_{\epsilon \epsilon^{\prime}}\left(\omega_{k}\right)$ with $\epsilon, \epsilon^{\prime}= \pm 1$ denoting the matrix element from wave number $\epsilon^{\prime} k$ to $\epsilon k$. If the interface has ideal right-hand chirality, there will be no phase shift for the uncoupled leftmoving photons $\left(S_{--}=1\right)$, whereas a $\pi$ phase shift is attained for the perfectly coupled right-moving photons $\left(S_{++}=-1\right)$. This phase difference of $\pi$ implies that the Mach-Zehnder interferometer, shown in Fig. 1(c), can be balanced such that a right-moving photon incident in port 1 exits through port 3 , whereas a left-moving photon incident in port 3 exits through port 2 , realizing a circulator. We define the fidelity of the circulator as the product of the probabilities for these two inputs to exit through the correct port, which is given by

$$
F_{R / L}(\omega)=\frac{1}{16}\left|1 \mp S_{++}(\omega)\right|^{2}\left|1 \pm S_{--}(\omega)\right|^{2} .
$$

Obviously $F_{R / L}=1$ if $S_{++}=\mp 1$ and $S_{--}= \pm 1$. In the Supplemental Material [39] we show the system Hamiltonian and use the input-output formalism to derive the scattering matrix, based on which the fidelity (2) can be numerically evaluated for given setups.

The bi-CPB core.-TRS can be broken by Josephson junction rings threaded by magnetic flux [24]. Here we use 
an equivalent "bi-CPB core" [39] depicted in the green box of Fig. 1(a). It has two CPBs denoted by " $a$ " and " $b$," which are assumed identical for now. Each CPB has a junction with Josephson energy $E_{J}$ and capacitance $C_{J}$. The voltage source determines the offset charge $n_{q, a / b}$ through a capacitor with capacitance $C_{g}$. We define $C_{\Sigma}=C_{J}+C_{g}$. The two CPBs are connected by a Josephson junction with capacitance $C_{a b}$ and Josephson energy $E_{J, a b}$. The external flux $\varphi_{\text {ext }}$ is made dimensionless by expressing it in units of $\Phi_{0} /(2 \pi)$ with $\Phi_{0}$ being the flux quantum. As depicted in Fig. 1(b), the transmission line waveguide is modeled by an infinite chain of coupled LC oscillators with capacitance $c \delta_{z}$ and inductance $l \delta_{z}$. The waveguide parameters are set by realistic impedance $Z=\sqrt{l / c}=50 \Omega$ and the speed of light $v_{g}=1 / \sqrt{l c} \approx 1.2 \times 10^{8} \mathrm{~m} / \mathrm{s}$. The elementary length, $\delta_{z}$, is determined by the lateral size of the coupling capacitor $C_{c}$ (or $C_{q}$ if using only the bi-CPB core).

At first, we consider coupling the bi-CPB core directly to the waveguide at $x_{l / r}$, with device parameters given in the caption of Fig. 2. Particularly, we set $c \delta_{z}=1 \mathrm{fF}$ corresponding to $\delta_{z} \approx 5 \mu \mathrm{m}$, which is comparable to the size of typical CPBs [25]. To suppress the dephasing caused by charge noise, the offset charge $n_{g, a / b}$ is fixed at the "sweet spot" where $\partial \omega_{01} / \partial n_{g}=0$ [40] and $\omega_{0 n}$ is the energy gap between the $n$th excited state and the ground state of the core (the sweet spot $n_{g, a / b}=0.5$ must be excluded, because here particle-hole symmetry preserves TRS). The separation $d=x_{r}-x_{l}$ is selected so that $k_{0} d=\pi / 2$.

In Fig. 2(a), we plot $F_{L}(\omega)$ as a function of detuning from $\omega_{01}$ for $\varphi_{\text {ext }}=0.5 \pi$ and a few values of $E_{J, a b}$. The plot shows that the bare core can indeed act as a chiral emitter, but suffers from several shortcomings. First, the peak fidelity, $\max _{\omega} F_{R / L}(\omega)$, is sensitive to the exact value of $E_{J, a b}$, leaving the device performance highly sensitive to fabrication variation. Fabrication variations might be compensated by adapting the tunable flux $\varphi_{\text {ext }}$. To examine this, we choose a representative $E_{J, a b}=5.5 h \mathrm{GHZ}$ corresponding to a $10 \%$ deviation from the ideal value and plot $F_{L}(\omega)$ as a function of $\varphi_{\text {ext }}$ in Fig. 2(b). It shows that this tuning fails to make the maximal fidelity above 0.9 . Furthermore, tuning $\varphi_{\text {ext }}$ changes $\omega_{01}$, rendering this configuration inadequate for tasks where photon frequencies are fixed. Figure 2 also shows that the bandwidth is less than $1 \mathrm{MHz}$, which severely limits the functionality.

The transmon-coupled bi-CPB chiral photonic interface.-The bandwidth is limited by the weak interaction between the small $\mathrm{CPBs}$ and the waveguide excitations delocalized on wavelength scale. To overcome this, we propose to insert transmons, which have lateral size in between the CPBs and the photonic wavelength, as efficient mediators. Compared with similar proposals using microwave resonators [27,29], transmons allow for stronger spatial confinement of excitations, and thereby larger couplings to the $\mathrm{CPBs}$, while simultaneously maintaining

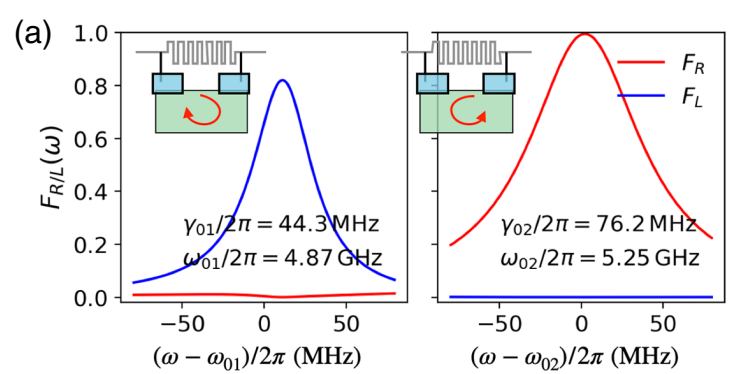

(b)

(c)

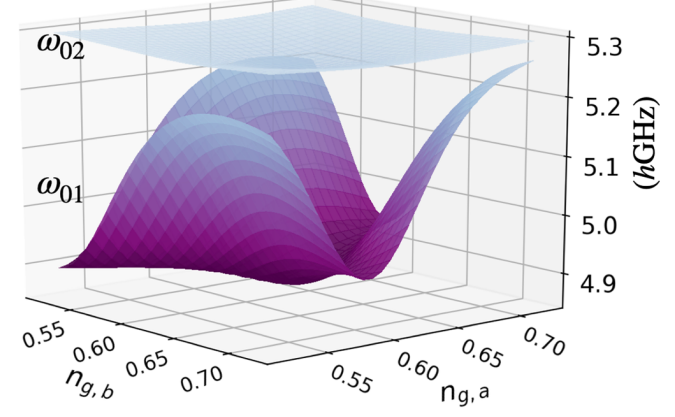

\begin{tabular}{ccc|cccccc|ccc}
\hline \hline$c \delta_{z}$ & $k_{0} d$ & $C_{q}$ & $C_{\Sigma}$ & $C_{a b}$ & $E_{J}$ & $E_{J, a b}$ & $\varphi_{\mathrm{ext}}$ & $C_{c}$ & $C_{t}$ & $E_{J} t$ \\
\hline 50 & $0.5 \pi$ & 1 & 1 & 1 & 5.5 & 5 & $0.5 \pi$ & 100 & 30 & 13 \\
\hline
\end{tabular}

\begin{tabular}{llllllllllll}
\hline 50 & $0.5 \pi$ & 1 & 1 & 1 & 5.5 & 5 & $0.5 \pi$ & 100 & 30 & 13 \\
\hline
\end{tabular}

FIG. 3. Energy levels and performance of the full system. (a) Fidelity of circulator as a function of the detuning from $\omega_{01}$ and $\omega_{02}$ with device parameters in (c). (b) Values (in unit of $h \mathrm{GHz}$ ) of $\omega_{01}$ and $\omega_{02}$ of the full device as a function of $n_{g, a / b}$, using parameters listed in (c), where units for capacitances and Josephson energies are $\mathrm{fF}$ and $h \mathrm{GHz}$, respectively.

sizable coupling to waveguides. A similar advantage could be achieved with high impedance elements [29], but the transmons additionally allow wide tunability as discussed below. In Fig. 3(a) we plot $F_{R / L}(\omega)$ as a function of the detuning to the first two excited states $\left(\omega_{01}\right.$ and $\left.\omega_{02}\right)$ for the CPB-transmon-coupled system. The device parameters used in the calculation are listed in Fig. 3(c), which are chosen to be representative for typical experimental situations and not fully optimized. Particularly, we have taken $c \delta_{z}=50 \mathrm{fF}$, i.e., $\delta_{z} \approx 250 \mu \mathrm{m}$, corresponding to the lateral size of the transmon reported in Ref. [41]. Figure 3(a) shows that the bandwidth is significantly improved. However, while a left-hand chirality $\left(F_{L} \gg F_{R}\right)$ is realized for $\omega \approx \omega_{01}$ (and $\omega \approx \omega_{03}$ ) -similar to the bare bi-CPB core-we observe the opposite chirality for $\omega \approx \omega_{02}$ $\left(F_{R} \gg F_{L}\right)$. This feature indicates the distinctness of the 2nd excited state. Below we use an approximate model to highlight multiple benefits brought by this state.

We assume that the two transmons, indexed by " $A$ " and " $B$ " in Fig. 1(a), are identical in parameters, and consider a restricted Hilbert space spanned by the ground state and the singly excited states of either transmon $A, B$, or the bi-CPB core, which will be denoted by $\left|1_{A}\right\rangle,\left|1_{B}\right\rangle$, and $|e\rangle$, respectively. The device Hamiltonian reads $H=$ $H_{0}+H_{\text {int }}$, where $H_{0}=e_{1}|e\rangle\langle e|+\omega_{t}\left(\left|1_{A}\right\rangle\left\langle 1_{A}|+| 1_{B}\right\rangle\left\langle 1_{B}\right|\right)$ 
with $e_{1}$ and $\omega_{t}$ the energies of the 1 st excited states of the core and the transmons, respectively. The interaction Hamiltonian is

$$
H_{\mathrm{int}}=g \hat{n}_{A}\left(\hat{n}_{a}-n_{g, a}\right)+g \hat{n}_{B}\left(\hat{n}_{b}-n_{g, b}\right),
$$

where $g$ is the coupling strength. Using rotating-wave approximation upon $H_{\text {int }}$, and restricting it to the reduced Hilbert space, we rewrite $H$ as

$$
H=H_{0}+\tilde{g}\left(\left|\psi_{c}\right\rangle\langle e|+| e\rangle\left\langle\psi_{c}\right|\right),
$$

where $\tilde{g}$ is modified from $g$ and

$$
\left|\psi_{\mathrm{c}}\right\rangle \propto \eta_{a}^{*}\left|1_{A}\right\rangle+\eta_{b}^{*}\left|1_{B}\right\rangle,
$$

with $\eta_{a(b)}=\left\langle e\left|\hat{n}_{a(b)}\right| g_{a b}\right\rangle$ as in Eq. (1). The coupling between $\left|\psi_{c}\right\rangle$ and $|e\rangle$ yields the 1st excited state $\left|\Psi_{-}\right\rangle$ with energy $\omega_{01}=e_{1}+\Delta_{-}$and the 3 rd excited state $\left|\Psi_{+}\right\rangle$ with energy $\omega_{03}=e_{1}+\Delta_{+}$,

$$
\left|\Psi_{ \pm}\right\rangle \propto \underbrace{\Delta_{ \pm}\left|\psi_{\mathrm{c}}\right\rangle}_{\text {transmons }}+\underbrace{i \tilde{g}|e\rangle}_{\text {bi-CPB }},
$$

where $\Delta_{ \pm}=-\delta / 2 \pm \sqrt{\delta^{2} / 4+\tilde{g}^{2}}$ with $\delta=e_{1}-\omega_{t}$. The 2nd excited state is a so-called dark state [33], which has no excitation on the core,

$$
\left|\Psi_{\mathrm{de}}\right\rangle \propto \eta_{b}\left|1_{A}\right\rangle-\eta_{a}\left|1_{B}\right\rangle .
$$

Despite being decoupled, $\left|\Psi_{\mathrm{de}}\right\rangle$ still attains chiral property from the core. The constants $\eta_{a(b)}$, however, have different orders and signs in $\left|\Psi_{\mathrm{de}}\right\rangle$ and $\left|\psi_{c}\right\rangle$, which lead to opposite chirality.

A more important observation is that the state $\left|\Psi_{\mathrm{de}}\right\rangle$ does not contain the bi-CPB state $|e\rangle$. Thus, it is not affected by the $\mathrm{CPB}$ charge noise and keeps the charge noise insensitivity of the transmon qubits [27]. To illustrate this, Fig. 3(b) shows $\omega_{01}$ and $\omega_{02}$ as a function of the offset charges $n_{g, a / b}$. The results are numerically evaluated from the complete Hamiltonian (see Ref. [39] for details) using device parameters listed in Fig. 3(c). Figure 3(b) demonstrates that $\omega_{02}$ is significantly more stable than $\omega_{01}$. The fluctuation of $\omega_{02}$ does not exceed its linewidth $\gamma_{02} / 2 \pi \approx$ $76 \mathrm{MHz}$ despite the large variations in $n_{g, a / b}$. Thus, the offset charges $n_{g, a(b)}$ need not be at the sweet spots, and can be used as control knobs to provide tunability.

Tunability of the chiral interface.-First, we demonstrate that by tuning $n_{g, a / b}$ and $\varphi_{\text {ext }}$ we can address issues of fabrication variation in the device parameters. To simulate realistic fabrication variations, we sample values of capacitance and CPB Josephson energy uniformly in intervals bounded by $\pm 1 \%$ and $\pm 10 \%$, respectively, from the intended values listed in Fig. 3(c). The transmon
Josephson energy $E_{J t}$ is fixed at the intended values because they can be tuned in situ via flux control. We collect 150 such "realistic samples," which includes cases with asymmetries between CPB-a and CPB-b, and between transmon- $A$ and transmon- $B$. In Fig. 4(a), we fix $n_{g, a / b}$ and $\varphi_{\text {ext }}$ at the optimal value for the ideal parameters, and show by gray points their individual maximal fidelity (vertical coordinate), frequency where the maximal fidelity is obtained (horizontal coordinate), and the $F_{R / L}>0.9$ bandwidth (horizontal error bar). While the performance is generally good, certain instances with poor chiral fidelity are observed. Next, for each sample that has a maximal fidelity less than 0.99 , we search for values of $n_{g, a / b}$ and $\varphi_{\text {ext }}$ that improve the performance. The red points in Fig. 4(a) show the result of such optimization. We are always able to tune the maximal fidelity above 0.99 . Additional details about how the maximal fidelity and bandwidth depend on $n_{g, a / b}$ and $\varphi_{\text {ext }}$ are given in the Supplemental Material [39].

Additionally, flux-tunable transmons [27] enables tuning of the operating frequency of our device. We consider the parameters listed in Fig. 3(c) (including the separation $d$ ). For 100 values of $E_{J t}$ between $6 \sim 22 \mathrm{hGHz}$, we optimize the peak fidelity to be higher than 0.99 by tuning $n_{g, a / b}$ and $\varphi_{\text {ext }}$. The results are presented in Fig. 4(b). It shows that for photons in a band of $3 \mathrm{GHz}$ width, the same device can always be tuned to provide an excellent chiral interaction. This broad adaptability is rooted in the breaking of TRS. When we tune $E_{J t}, \omega_{02}$ changes and so does the phase $k_{0} d$
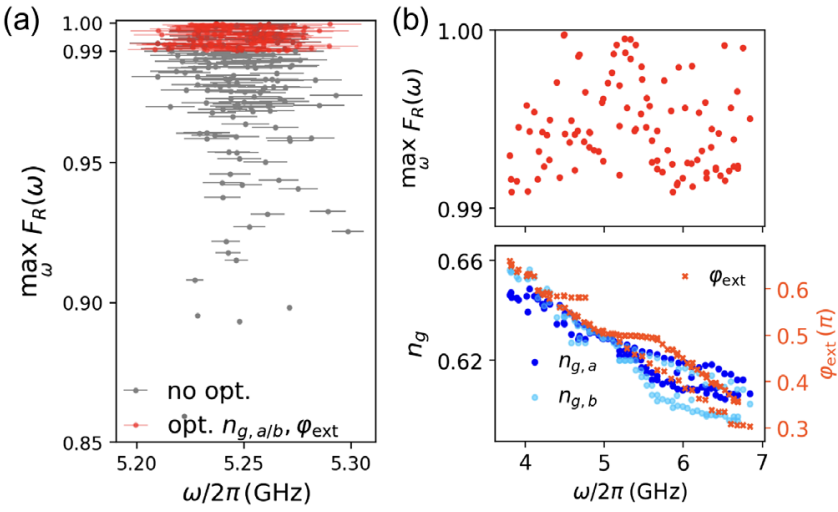

FIG. 4. Tunability of the chiral interface. (a) 150 realizations of the chiral interfaces with device parameters randomly sampled to represent fabrication variations (see text for details). The marker shows peak fidelity for a given realization, error bar indicates the $F_{R}>0.9$ bandwidth. The gray points show results obtained when $n_{g, a / b}$ and $\varphi_{\text {ext }}$ are optimized for the ideal parameters listed in Fig. 3(c). Red points show fidelities obtained when $n_{g, a / b}$ and $\varphi_{\text {ext }}$ are optimized for each realization. (b) Upper panel: Peak fidelities as a function of photon frequency, for the chiral interface with parameters given in Fig. 3(c) and 100 samples of transmon Josephson energy $E_{J t}$ between $6 \sim 22 \mathrm{hGHz}$. For each red dot thereof, the vertically aligned markers in the lower panel shows the adaptively optimized $n_{g, a / b}$ and $\varphi_{\text {ext }}$. 
with $k_{0}=\omega_{02} / v_{g}$. Adjusting the offset charges and flux, however, allows us to achieve complete destructive interference in one of the directions. Our chiral interface thus works in principle as long as $k_{0} d \neq 0(\bmod \pi)$.

Discussion and conclusion.-The bandwidth for $F_{R / L}(\omega)>0.9$ is about $25 \mathrm{MHz}$ in Fig. 3(a). This is limited by the transmon decay rate, which is typically on the order of $100 \mathrm{MHz}$. The bandwidth can thus be directly enhanced by designing transmons with larger decay rates. We note, however, that as the decay rate approaches roughly a tenth of the transition frequency $\left(0.1 \omega_{0} / 2 \pi \sim 500 \mathrm{MHz}\right)$ the system reaches the ultrastrong coupling regime of waveguide QED [42] requiring a more advanced theoretical description [43,44].

Our interface relies on the 2 nd excited state. This raises the concern that decay to the 1st excited state may jeopardize the performance. This transition is negligible for two reasons. First, the transition matrix element is tiny (vanishing in our simplified model $\left\langle\Psi_{-}\left|\hat{n}_{A / B}\right| \Psi_{\mathrm{de}}\right\rangle=0$ ). Second, the waveguide mode density at $\omega_{12} / 2 \pi=\left(\omega_{02}-\omega_{01}\right) / 2 \pi \sim$ $100 \mathrm{MHz}$ is low. As a result, the decay rate to the 1 st excited state is numerically found to be $\gamma_{12} / 2 \pi \sim 10 \mathrm{kHz}$, which is much smaller than $\gamma_{02} / 2 \pi \sim 100 \mathrm{MHz}$. Similarly, the intrinsic loss of the components involved can be made well below $0.1 \mathrm{MHz}[34,45]$. This is much less than the decay rate to the waveguide and hence negligible [39].

In conclusion, we have shown that by introducing two transmons, a passive charge-noise insensitive chiral photonic interface can be built, based on a core consisting of $\mathrm{CPBs}$ and an external magnetic flux that breaks TRS. The interface has rich tunability, making it tolerant to experimentally relevant fabrication variations. The bandwidth of $F_{R / L}>0.9$ is a few tens of $\mathrm{MHz}$ for realistic transmon parameters with decay rates typically around $100 \mathrm{MHz}$. With tunable transmons (SQUIDs), our design can be tuned in a band as wide as a few GHz. The lateral dimension of the entire circulator can be a few millimeters [39]. These properties make our chiral interface highly attractive for, e.g., on-chip routing of microwave signals $[10,14-23,36,37]$ as well as fundamental studies of chiral waveguide QED [3,4,7,46].

A. S. S. thanks Peter Lodahl, Per Delsing, and Gran Johansson for useful discussions. M. K. gratefully acknowledges helpful and insightful discussions with Bharath Kannan. Y.-X.Z. and A.S. S. thank Jacob M. Taylor and Brittany Richman for insightful discussions. Y.-X. Z., M. K., and A. S. S. acknowledge financial support from the Danish National Research Foundation. M. Kjaergaard was also supported by Villum Fonden (Grant No. 37467) through a Villum Young Investigator grant.

*iyxz@nbi.ku.dk

[1] C. W. Gardiner, Phys. Rev. Lett. 70, 2269 (1993).
[2] H. J. Carmichael, Phys. Rev. Lett. 70, 2273 (1993).

[3] T. Ramos, H. Pichler, A. J. Daley, and P. Zoller, Phys. Rev. Lett. 113, 237203 (2014).

[4] I. M. Mirza and J. C. Schotland, Phys. Rev. A 94, 012302 (2016).

[5] J.-T. Shen and S. Fan, Phys. Rev. A 76, 062709 (2007).

[6] H. Zheng, D. J. Gauthier, and H. U. Baranger, Phys. Rev. Lett. 107, 223601 (2011).

[7] S. Mahmoodian, G. Calajó, D. E. Chang, K. Hammerer, and A. S. Sørensen, Phys. Rev. X 10, 031011 (2020).

[8] V. I. Yudson, Zh. Eksp. Teor. Fiz. 88, 1757 (1985) [Sov. Phys. JETP 61, 1043 (1985)], http://83.149.229.155/cgibin/e/index/e/61/5/p1043?a=list.

[9] P. Lodahl, S. Mahmoodian, S. Stobbe, A. Rauschenbeutel, P. Schneeweiss, J. Volz, H. Pichler, and P. Zoller, Nature (London) 541, 473 (2017).

[10] I.-C. Hoi, C. M. Wilson, G. Johansson, T. Palomaki, B. Peropadre, and P. Delsing, Phys. Rev. Lett. 107, 073601 (2011).

[11] A. Blais, A. L. Grimsmo, S. M. Girvin, and A. Wallraff, Rev. Mod. Phys. 93, 025005 (2021).

[12] D. Roy, C. M. Wilson, and O. Firstenberg, Rev. Mod. Phys. 89, 021001 (2017).

[13] C. Caloz, A. Alù, S. Tretyakov, D. Sounas, K. Achouri, and Z.-L. Deck-Léger, Phys. Rev. Applied 10, 047001 (2018).

[14] K. M. Sliwa, M. Hatridge, A. Narla, S. Shankar, L. Frunzio, R. J. Schoelkopf, and M. H. Devoret, Phys. Rev. X 5, 041020 (2015).

[15] B. Abdo, K. Sliwa, S. Shankar, M. Hatridge, L. Frunzio, R. Schoelkopf, and M. Devoret, Phys. Rev. Lett. 112, 167701 (2014).

[16] B. Abdo, N. T. Bronn, O. Jinka, S. Olivadese, A. D. Córcoles, V. P. Adiga, M. Brink, R. E. Lake, X. Wu, D. P. Pappas, and J. M. Chow, Nat. Commun. 10, 3154 (2019).

[17] J. Kerckhoff, K. Lalumière, B. J. Chapman, A. Blais, and K. W. Lehnert, Phys. Rev. Applied 4, 034002 (2015).

[18] S. Barzanjeh, M. Wulf, M. Peruzzo, M. Kalaee, P. B. Dieterle, O. Painter, and J. M. Fink, Nat. Commun. 8, 953 (2017).

[19] A. Metelmann and H. E. Türeci, Phys. Rev. A 97, 043833 (2018).

[20] A. Kamal, J. Clarke, and M. H. Devoret, Nat. Phys. 7, 311 (2011).

[21] N. A. Estep, D. L. Sounas, J. Soric, and A. Alù, Nat. Phys. 10, 923 (2014).

[22] P. Roushan et al., Nat. Phys. 13, 146 (2017).

[23] B. J. Chapman, E. I. Rosenthal, and K. W. Lehnert, Phys. Rev. Applied 11, 044048 (2019).

[24] J. Koch, A. A. Houck, K. L. Hur, and S. M. Girvin, Phys. Rev. A 82, 043811 (2010).

[25] A. Wallraff, D. I. Schuster, A. Blais, L. Frunzio, R.-S. Huang, J. Majer, S. Kumar, S. M. Girvin, and R. J. Schoelkopf, Nature (London) 431, 162 (2004).

[26] C. Müller, S. Guan, N. Vogt, J. H. Cole, and T. M. Stace, Phys. Rev. Lett. 120, 213602 (2018).

[27] J. Koch, T. M. Yu, J. Gambetta, A. A. Houck, D. I. Schuster, J. Majer, A. Blais, M. H. Devoret, S. M. Girvin, and R. J. Schoelkopf, Phys. Rev. A 76, 042319 (2007). 
[28] C. R. i Carceller, Master's thesis, University of Copenhagen, 2020.

[29] B. Richman and J. M. Taylor, PRX Quantum 2, 030309 (2021).

[30] A. Frisk Kockum, P. Delsing, and G. Johansson, Phys. Rev. A 90, 013837 (2014).

[31] A. F. Kockum, in Proceedings of the International Symposium on Mathematics, Quantum Theory, and Cryptography, edited by T. Takagi, M. Wakayama, K. Tanaka, N. Kunihiro, K. Kimoto, and Y. Ikematsu (Springer, Singapore, 2021).

[32] B. Kannan, M. J. Ruckriegel, D. L. Campbell, A. F. Kockum, J. Braumüller, D. K. Kim, M. Kjaergaard, P. Krantz, A. Melville, B. M. Niedzielski, A. Vepsäläinen, R. Winik, J. L. Yoder, F. Nori, T. P. Orlando, S. Gustavsson, and W. D. Oliver, Nature (London) 583, 775 (2020).

[33] E. Arimondo and G. Orriols, Lett. Nuovo Cimento17, 333 (1976).

[34] A. P. M. Place, L. V. H. Rodgers, P. Mundada, B. M. Smitham, M. Fitzpatrick, Z. Leng, A. Premkumar, J. Bryon, A. Vrajitoarea, S. Sussman, G. Cheng, T. Madhavan, H. K. Babla, X. H. Le, Y. Gang, B. Jäck, A. Gyenis, N. Yao, R. J. Cava, N. P. de Leon, and A. A. Houck, Nat. Commun. 12, 1779 (2021).

[35] M. Kjaergaard, M. E. Schwartz, J. Braumüller, P. Krantz, J. I.-J. Wang, S. Gustavsson, and W. D. Oliver, Annu. Rev. Condens. Matter Phys. 11, 369 (2020).
[36] P.-O. Guimond, B. Vermersch, M. L. Juan, A. Sharafiev, G. Kirchmair, and P. Zoller, npj Quantum Inf. 6, 32 (2020).

[37] N. Gheeraert, S. Kono, and Y. Nakamura, Phys. Rev. A 102, 053720 (2020).

[38] X. Wang and H.-r. Li, arXiv:2106.13187.

[39] See Supplemental Material at http://link.aps.org/supplemental/ 10.1103/PhysRevLett.127.233601 for detailed derivations and additional plots.

[40] D. Vion, Science 296, 886 (2002).

[41] M. Mirhosseini, E. Kim, X. Zhang, A. Sipahigil, P. B. Dieterle, A. J. Keller, A. Asenjo-Garcia, D. E. Chang, and O. Painter, Nature (London) 569, 692 (2019).

[42] P. Forn-Díaz, J. J. García-Ripoll, B. Peropadre, J.-L. Orgiazzi, M. A. Yurtalan, R. Belyansky, C. M. Wilson, and A. Lupascu, Nat. Phys. 13, 39 (2017).

[43] E. Sanchez-Burillo, D. Zueco, J. J. Garcia-Ripoll, and L. Martin-Moreno, Phys. Rev. Lett. 113, 263604 (2014).

[44] T. Shi, Y. Chang, and J. J. García-Ripoll, Phys. Rev. Lett. 120, 153602 (2018).

[45] R. Barends, J. Kelly, A. Megrant, D. Sank, E. Jeffrey, Y. Chen, Y. Yin, B. Chiaro, J. Mutus, C. Neill, P. O’Malley, P. Roushan, J. Wenner, T. C. White, A. N. Cleland, and J. M. Martinis, Phys. Rev. Lett. 111, 080502 (2013).

[46] S. Mahmoodian, M. Čepulkovskis, S. Das, P. Lodahl, K. Hammerer, and A. S. Sørensen, Phys. Rev. Lett. 121, 143601 (2018). 